\title{
ALMOST CONTINUOUS EXTENSION FOR TAUT FOLIATIONS
}

\author{
DANny CALEgari
}

\begin{abstract}
A taut foliation $\mathscr{F}$ of a hyperbolic 3 -manifold $M$ has the continuous extension property for leaves in almost every direction; that is, for each leaf $\lambda$ of $\widetilde{F}$ and almost every geodesic ray $\gamma$ in $\lambda$ the limit of $\gamma$ in $\widetilde{M}$ is a well-defined point in the ideal boundary of $\widetilde{M}=\mathbb{H}^{3}$.
\end{abstract}

\section{Introduction}

Let $\mathscr{F}$ be a taut foliation of an atoroidal 3-manifold $M$. Then a theorem of Candel ([1]) says that there is a path metric on $M$ such that with their induced path metrics, leaves of $\mathscr{F}$ are locally isometric to $\mathbb{H}^{2}$. In particular, it follows that for any metric on $M$, the leaves of $\widetilde{\mathscr{F}}$ with their induced path metrics are uniformly quasi-isometric to $\mathbb{H}^{2}$, and therefore have a well-defined circle at infinity. For a leaf $\lambda$ of $\widetilde{\mathscr{F}}$ we denote this circle at infinity by $S_{\infty}^{1}(\lambda)$. Actually, one only needs to know leaves of $\widetilde{\mathscr{F}}$ are quasi-isometric to hyperbolic planes to construct these circles at infinity, a fact which is much easier to prove than Candel's theorem.

If $M$ is hyperbolic, there is an identification $\widetilde{M}=\mathbb{H}^{3}$ and there is a natural ideal boundary which we denote by $S_{\infty}^{2}$.

A basic problem in the theory of foliations of 3-manifolds is to understand the relationship between the intrinsic geometry of the leaves of the foliation and the extrinsic (coarse) geometry of the ambient manifold (usually in the universal cover). In particular, a question that has received a lot of attention has been the following

Question 1. Let $\mathscr{F}$ be a taut foliation of a hyperbolic 3-manifold. Does the inclusion $i: \lambda \rightarrow \widetilde{M}=\mathbb{H}^{3}$ extend continuously to a map on the ideal boundaries $i_{\partial}: S_{\infty}^{1}(\lambda) \rightarrow S_{\infty}^{2}\left(\mathbb{H}^{3}\right)$ ?

This is a difficult question, and the (positive) answer is only known in certain cases. In particular, the answer is known for surface bundles over the circle ([2]), for depth one and certain other finite depth taut foliations ([3],[4]) and other special cases. The problem is that leaves of taut foliations are far from being quasi-isometrically embedded in the universal cover, so a path which is quasi-geodesic in a leaf may potentially fail to limit to a definite point in the

Received November 16, 2000. 
ideal boundary of the ambient space. The subtlety of the question is evidenced by the complicated structure of the image of such $i_{\partial}$; limit sets of leaves are frequently "exotic" geometric sets such as dendrites, gaskets or sphere-filling curves.

It is easy to see, from the properness of leaves of $\widetilde{\mathscr{F}}$, that if $i_{\partial}$ is defined for each point then it is continuous. It turns out that if we only want to show that $i_{\partial}$ is defined almost everywhere, then there is a surprisingly simple proof, which works immediately for all taut foliations of hyperbolic 3-manifolds.

Theorem 1. With notation as above, for every leaf $\lambda$ of $\widetilde{\mathscr{F}}$, for almost all geodesic rays $r \subset \lambda$, the ray $r \subset \mathbb{H}^{3}$ converges to a definite point in $S_{\infty}^{2}$, and defines a measurable map $i_{\partial}: S_{\infty}^{1}(\lambda) \rightarrow S_{\infty}^{2}\left(\mathbb{H}^{3}\right)$.

Proof. Actually, the only property of a taut foliation we use is that $\lambda$ is quasiisometric to $\mathbb{H}^{2}$ and the embedding $i: \lambda \rightarrow \mathbb{H}^{3}$ is proper with bounded geometry and extends to a collar neighborhood of $\lambda$. That is, there is an $\epsilon$ so that there is a quasi-isometric embedding $I: \lambda \times[-\epsilon, \epsilon] \rightarrow \mathbb{H}^{3}$ such that $I(p, 0)=i(*)$.

That the geometry of the embedding is bounded follows from the compactness of $M$. To see that there is a uniform collar neighborhood of $i(\lambda)$, observe that there is a uniform $\delta$ so that the $\delta$-neighborhood of any point in $M$ is contained in a ball foliated as a product. If $\lambda$ were to intersect such a lift of a product ball in two distinct disks in $\widetilde{M}$ we could find a transversal $\tau$ to $\widetilde{\mathscr{F}}$ from $\lambda$ to itself, and therefore by perturbation, a closed loop $\gamma \subset \widetilde{M}$ transverse to $\widetilde{\mathscr{F}}$. This contradicts the well-known fact that for a taut foliation of a 3-manifold, transverse loops are homotopically essential.

Pick a basepoint $a \in \mathbb{H}^{3}$ through which the basepoint $p$ of $\lambda$ passes, and let $S$ be the visual sphere of $a$. There is an obvious visual projection $\pi: \mathbb{H}^{3}-a \rightarrow S$ which is basically just a version of the Gauss map for hyperbolic space. Let $B$ be the ball of radius 1 about $a$.

For a point $b \in \mathbb{H}^{3}$ with $\operatorname{dist}_{\mathbb{H}^{3}}(a, b)=t$ and a vector $v \in U T_{b} \mathbb{H}^{3}$ the norm $|d \pi(v)|$ is $O\left(e^{-t}\right)$. The area of a sphere of radius $t$ in $\mathbb{H}^{3}$ is $O\left(e^{2 t}\right)$. Therefore we can estimate

$$
\int_{\mathbb{H}^{3}-B}\|d \pi(x)\|^{\alpha} d \operatorname{vol}_{\mathbb{H}^{3}} \leq \int_{1}^{\infty} \text { const. } \cdot e^{t(2-\alpha)} d t<\infty \text { when } \alpha>2
$$

where $\|\cdot\|$ denotes the operator norm on $d \pi: T \mathbb{H}^{3} \rightarrow T S$.

Since $I$ is an embedding, we obviously have

$$
\int_{I(\lambda \times[-\epsilon, \epsilon]) \backslash B}\|d \pi(x)\|^{\alpha} d \operatorname{vol}_{\mathbb{H}^{3}}<\infty
$$

with the same assumption on $\alpha$, namely that $\alpha>2$.

The value of $\|d \pi(x)\|$ depends only on the distance from $x$ to $a$. So, away from $B$, the fact that $I$ is a quasi-isometry implies that this value varies only a bounded amount over $q \times[-\epsilon, \epsilon]$ for each $q \in \lambda \backslash B$ independently of $q$, and so 
there is a bounded cost in replacing the integral of this value over the interval with the value at $q \times 0$.

Moreover, we know $d I$ has uniformly bounded distortion over $\lambda$, by the comments above, so that in particular $d \mathrm{vol}_{\lambda} d t \leq$ const. $^{*} d \mathrm{vol}_{\mathbb{H}^{3}}$. Thus

$$
\int_{\lambda \backslash B}\|d \pi \circ i(x)\|^{\alpha} d \mathrm{vol}_{\lambda}<\infty
$$

In particular, using spherical co-ordinates on $\lambda$, we can conclude that for almost every geodesic ray $\gamma \subset \lambda$ emanating from $p$,

$$
\int_{c}^{\infty}\left|\frac{d \pi(\gamma(t))}{d t}\right|^{\alpha} e^{t} d t<\infty
$$

so in particular, $e^{t / \alpha} \frac{d \pi(\gamma(t))}{d t}$ is in $L^{\alpha}$. On the other hand, $e^{-t / \alpha}$ is certainly in $L^{\alpha /(\alpha-1)}$, so by Hölder's inequality $\frac{d \pi(\gamma(t))}{d t}$ is in $L^{1}$. That is, the position of $\pi(\gamma(t))$ in $S$ moves only a bounded amount and therefore has a well-defined limit. (Here we have chosen a hyperbolic metric on $\lambda$ according to Candel's theorem; such a metric is quasi-isometric to the path metric on $\lambda$ inherited as a subspace of $\mathbb{H}^{3}$.)

It remains to show that $i_{\partial}$ is measurable. Recall that $\pi: \mathbb{H}^{3}-a \rightarrow S$ is just radial projection onto the visual sphere at $a$. Let $\phi: S \rightarrow S_{\infty}^{2}$ be the visual identification. For any positive real number $r$, let $S_{r}(p)$ denote the circle of radius $r$ in $\lambda$ centered at $p$, and let

$$
f_{r}: S_{r}(p) \rightarrow S_{\infty}^{1}(\lambda)
$$

be radial projection in $\lambda$. Then

$$
\phi \pi f_{r}^{-1}: S_{\infty}^{1}(\lambda) \rightarrow S_{\infty}^{2}
$$

is continuous for each $r$, and converges pointwise a.e. to $i_{\partial}$ as $r \rightarrow \infty$. In particular, this limit is measurable.

Remark 1. Basically the point of the proof is the following: hyperbolic $3^{-}$ space grows in volume like $O\left(e^{2 t}\right)$; a leaf of a taut foliation grows in area like $O\left(e^{k t}\right)$ where $-k$ is the "coarse" negative curvature on a large scale of the leaf with respect to the induced subspace metric (obviously $1 \leq k \leq 2$ and can be estimated from a quasi-isometry constant of a uniformizing map $\lambda \rightarrow \mathbb{H}^{2}$ ). Since the embedding of the leaf in $\mathbb{H}^{3}$ is a quasi-isometry into its $\epsilon$-neighborhood for some $\epsilon$, the volume of a collar neighborhood of the leaf can be efficiently measured by its area. Since the growth rate of both $\lambda$ and $\mathbb{H}^{3}$ are exponential, it follows that "most" paths in $\lambda$ make roughly comparable progress in $\mathbb{H}^{3}$ and in $\lambda$; that is, "most" quasigeodesic rays in $\lambda$ limit to a definite point in $S_{\infty}^{2}$.

Remark 2. A more subtle analysis of the properties of proper embedded minimal planes in $\mathbb{H}^{3}$ shows that we can actually estimate $k \leq \sqrt{2}$ (see [6]). 
Remark 3. The proof applies essentially without modification to show that leaves of the universal covers of essential laminations have measurable extensions to $S_{\infty}^{2}$. The only technical issues are, firstly, that essential laminations do not admit homotopically trivial tight transverse loops (by [5]), and secondly that the leaves of the universal cover of an essential lamination of a hyperbolic manifold, with their induced path metrics, are uniformly quasi-isometric to hyperbolic planes. This follows from Candel's theorem.

Acknowledgement. I would like to thank the referee for some useful comments.

\section{References}

[1] A. Candel, Uniformization of surface laminations, Ann. Sci. École Norm. Sup. (4) 26 (1993), no.4, 489-516.

[2] J. Cannon and W. Thurston, Group invariant Peano curves, preprint circa 1985.

[3] S. Fenley, Asymptotic properties of depth one foliations in hyperbolic 3-manifolds, J. Differential Geom. 36 (1992), no.2, 269-313.

[4] S. Fenley, Foliations with good geometry, J. Amer. Math. Soc. 12 (1999), no.3, 619-676.

[5] D. Gabai and U. Oertel, Essential laminations in 3-manifolds, Ann. of Math. (2) 130 (1989) no. 2, 41-73.

[6] W. Thurston, Three-manifolds, foliations and circles, II, preprint circa 1997.

Department of Mathematics, Harvard, CAmbridge, MA 02138.

E-mail address: dannyc@math.harvard.edu 